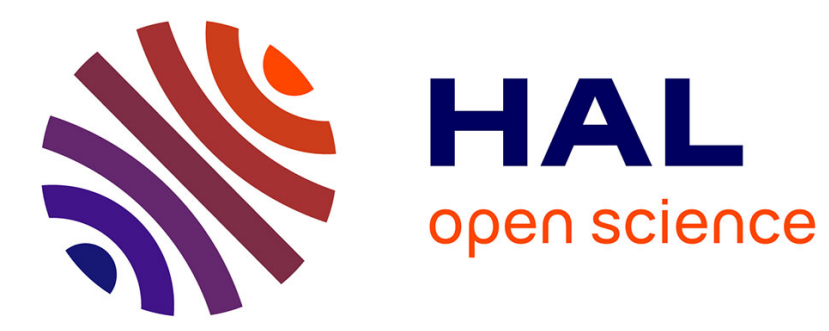

\title{
Complexity analysis of experimental cardiac arrhythmia
}

\author{
Binbin Xu, Stéphane Binczak, Sabir Jacquir, Oriol Pont, Hussein Yahia
}

\section{To cite this version:}

Binbin Xu, Stéphane Binczak, Sabir Jacquir, Oriol Pont, Hussein Yahia. Complexity analysis of experimental cardiac arrhythmia. IEEE TENSYMP 2014, IEEE, Apr 2014, Kuala Lumpur, Malaysia. hal-00919514v2

\section{HAL Id: hal-00919514 \\ https://inria.hal.science/hal-00919514v2}

Submitted on 31 Jan 2014

HAL is a multi-disciplinary open access archive for the deposit and dissemination of scientific research documents, whether they are published or not. The documents may come from teaching and research institutions in France or abroad, or from public or private research centers.
L'archive ouverte pluridisciplinaire HAL, est destinée au dépôt et à la diffusion de documents scientifiques de niveau recherche, publiés ou non, émanant des établissements d'enseignement et de recherche français ou étrangers, des laboratoires publics ou privés. 


\title{
Complexity analysis of experimental cardiac arrhythmia
}

\author{
Binbin $\mathrm{Xu}^{1}$, Stéphane Binczak ${ }^{2}$, Sabir Jacquir ${ }^{2}$, Oriol Pont ${ }^{1}$, Hussein Yahia ${ }^{1}$ \\ 1 GEOSTAT, INRIA Bordeaux Sud-Ouest, Talence, France \\ 2 CNRS UMR 6306, LE2I Université de Bourgogne, Dijon France
}

\begin{abstract}
To study the cardiac arrhythmia, an in vitro experimental model and Multielectrodes Array (MEA) are used. This platform serves as an intermediary of the electrical activities of cardiac cells and the signal processing / dynamics analysis. Through it the extracellular potential of cardiac cells is acquired, allowing a real-time monitoring / analyzing. Since MEA has 60 electrodes / channels dispatched in a rectangular region, it allows real-time monitoring and signal acquisition on multiple sites. The in vitro experimental model (cardiomyocytes cultures from new-born rats' heart) is directly prepared on the MEA. This carefully prepared culture has similar parameters as cell of human's heart. In order to discriminate the cardiac arrhythmia, complexity analysis methods (Approximate Entropy, ApEn and Sample Entropy, SampEn) are used especially taking into account noise. The results showed that, in case of arrhythmia, the ApEn and SampEn are reduced to about $50 \%$ of the original entropies. Both parameters could be used as factors to discriminate arrhythmia. Moreover, from a point of view of biophysics this decrease $50 \%$ of Entropy coincides with the bifurcation (periods, attractors etc.) in case of arrhythmia which have been reported previously. It supports once more the hypothesis that in case of cardiac arrhythmia, the heart entered into chaos which helps to better understand the mechanism of atrial fibrillation.
\end{abstract}

\section{Introduction}

Atrial fibrillation, the most frequent cardiac disease, constitutes an important impact factor for public health. The annual number of deaths due to cardiovascular disease will increase from 17 million in 2008 to 25 million in 2030 [1], even decades of work has been devoted in investigation of the mechanism of AF in order to find relevant treatment, more efforts are still needed. The electrocardiological signals from the heart are an excellent intermediary to study the heart's behaviors and pathological conditions. The Electrocardiogram has been used since years to identify heart diseases. But it provides only a global view of heart electrical activities. Exploration at tissue or cellular level is impossible. The in vitro experimental model, by bridging the gap between in vivo and numerical studies, represents always a tool 
of importance. Intracellular recording has a quite good temporal resolution. Patch clamp can measure precisely the ionic current, trans-membrane potential in a single cell. Since these methods are invasive and use only one cell which miss the collective effect, it is hard to interpret obtained results at organ level.

The Multielectrode arrays system using cardiac cultures can in this case provide better spatial-tempo resolution [2]. It, through which neural / electrocardiological signals are obtained or delivered, can serve as interfaces that connect cells to electronic circuitry. Compared to more traditional methods such as patch clamping, it allows setting up many controls within the same experiments. For example, using one electrode to stimulate, the others are used to acquire signals; simultaneously recording signals from multiple sites is possible, etc. Furthermore, in vitro arrays are non-invasive when compared to patch clamping because they do not require breaching of the cell membrane.

Since the normal signal and arrhythmic ones are similar, discriminating them one from other is difficult. Visual representation allows discriminating them. However, it is less efficient. It would be more beneficial to classifier these signals in an automatic way.

\section{Materials and Methods}

\subsection{Cardiomyocytes culture on Multielectrode array}

The monolayer cardiac culture is prepared with cardiomyocytes $(\mathrm{CM})$ from new-born Wisler rat $(1 \sim 4$ days, details of culture preparing in [3]). This experimental model allows reproducing a wide range of pathological conditions such as ischemia reperfusion, the radical stress or thermal shock, and any combination of these conditions [3]. It can also be used to study cardiac arrhythmia which has been confirmed previously. Cardiac arrhythmia type signals can be induced by anomalous conduction, erratic pacemaker driving [4,5], electrical stimulation [6-8] or more recently by hypothermia [9]. Furthermore, compared to other rat heart models, the engineered model in this study featured a mean period of action potential $0.50-0.72$ second in normal conditions. The culture beating $(83-120$ beats per minute, bpm) is similar to normal human heart, which is different from other similar models (normal rat heart beats at $600 \mathrm{bpm}$ ). Other physiological parameters of this model are quite similar to those in vivo as well.

The CM culture is prepared directly on Multielectrode array (MEA) allowing recording in real-time the extracellular field potential (EFP) of the culture. The MEA used here has 60 electrodes in a pattern 


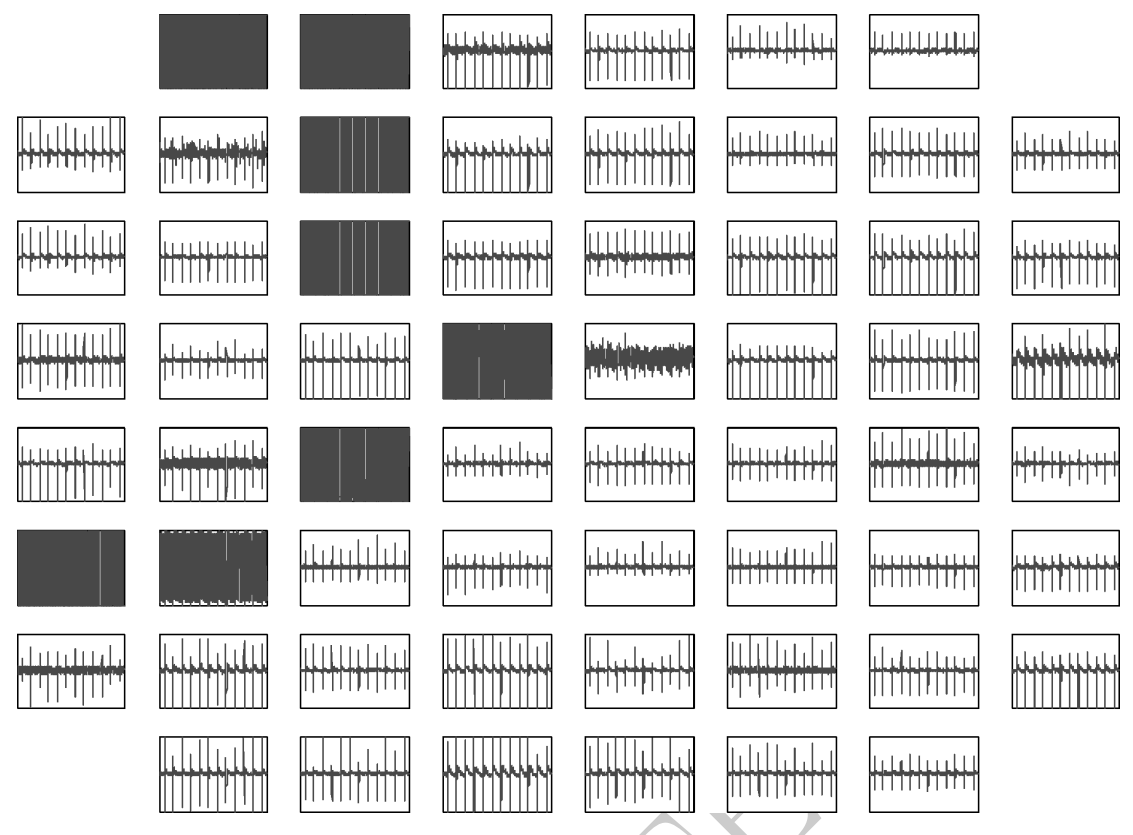

Figure 1. 60 EFP signals in basal conditions.

of $8 \times 8$ (Fig. 1 ). Electrodes are composed of titanium and have diameters $30 \mu \mathrm{m}$ and inter-electrodes distance is $100 \mu \mathrm{m}$. So compared to typical cardiac cell (of maximum length $100 \mu \mathrm{m}$, diameter $15 \mu \mathrm{m}$ ), the MEA provides a quite good spatial resolution. Since the recorded signals with MEA have a close correlation of the depolarization and repolarizing phase of the action potentials (Fig. 2), this allows to interpret indirectly the results at "action potential propagation" level $[10,11]$.

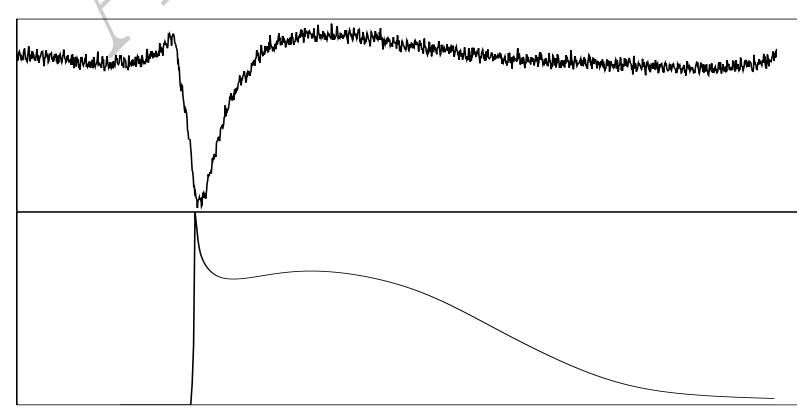

Figure 2. Schematic illustration of the correlation between the EFP signal (upper, real data) and the action potential (lower) 


\subsection{Signal denoising}

The acquired signals from the platform MEA are often disturbed by instruments noise (measure, processing algorithm of the system) and others environmental noises (electromagnetic interference, EMI) etc. To better process these signals and obtain more pertinent information, the first step is denoising. There exist many denoising methods. Here, we compared three methods in order to find the most suitable method to signal from the platform MEA.

Denoising by Savitzky-Golay filtering The smoothing filter Savitzky-Golay (smoothing polynomials or least squares) uses a local regression to determine the smoothed value for each point [12]. The main advantage of this approach is that it can preserve, in general, the characteristics of the distribution of

high frequencies such as maxima, minima and width, which are generally flattened by other standard techniques (such as moving averages, etc.). So this filter is quite popular in the processing of electrocardiological signals whose peaks must be preserved as fully as possible. Because EFP signals share some similarities with the ECG signals, the SGO filtering is the first method to test.

Denoising by Singular spectrum analysis The singular spectrum analysis is in practice a non-parametric estimation method (independent to models) [13]. The SSA is generally considered as a method of identification / extraction of oscillatory components in a signal. It allows obtaining key information of signal's spectra. The signal to treat is projected into space built with its eigenvectors. The relevant information is then reflected in the leading eigenvectors. Keeping the main components and eliminating the "noise" that are projected in other directions, it serves indeed as a denoising method.

Denoising by wavelet Numerous time-frequency methods are proposed to analyze the signals. Among them, the wavelet transform has been one of the most studied / used tools $[14,15]$. It is particularly useful because of its ability to simultaneously analyze a signal in both time and frequency using an observing window with variable width. The principle of wavelet denoising is the thresholding estimators [16]. If the energy of a signal is concentrated in a small number of wavelet dimensions, its coefficients will be relatively larger compared to those of other signals / noise. Finding an acceptable threshold, eliminating those smaller coefficients and performing an inverse wavelet transform, the original signal is then reconstructed / denoised. 


\subsection{Complexity analysis}

From system dynamics' view, the physiologic system is high-dimensional. According to Taken's theorem [17], it is possible to analyze this type of systems from available low-dimensional data (often one-dimensional time series measure). However, since the physiologic system is most likely affected by dynamical noise and perturbation, the approaches based on this theorem are deterministic and are then of limited use. Another type of methods is developed from stochastic approach which is aimed to quantify the statistical properties of the time series. Among them, the methods of complexity analysis are particularly useful to analyze time series in Electrocardiology in which the signals are characterized by their high regularity in normal condition in contrast to irregularity in pathological cases. Despite the fact that these time series most likely contain deterministic and stochastic components and both approaches may provide complementary information about the underlying dynamics, the former allows basically only a visual discrimination of normal case and pathological cases (methods such as phase space reconstruction $[17,18]$, recurrence plot $[19,20]$ etc.). Therefore, it is difficult to quantify the analysis. The methods based on complexity analysis are in general developed to quantify the degree of complexity of different time series. So, they are very useful to automate the discrimination process.

The approximate entropy (ApEn) reports on similarity in time series to test their regularity. It was initially developed to distinguish and quantify low-dimensional deterministic systems, periodic and multiply periodic systems, high-dimensional chaotic systems, stochastic and mixed systems from relatively short and noisy time series [21]. Signals with repetitive patterns of fluctuation are more predictable than those without repetitive patterns. ApEn can quantify the likelihood in time series. In general, a time series containing many repetitive patterns has a relatively small ApEn; a less predictable process has a higher ApEn. The wide use of ApEn in clinical cardiovascular studies has shown some very positive results.

However, since ApEn counts each sequence as matching itself, it produces biased estimation for selfmatching signal. In order to reduce this bias, Richman et al. improved the ApEn by proposing a new type Entropy estimation : Sample Entropy (SampEn) where self-matches are not included in calculating the probability [22]. 


$$
\begin{aligned}
\text { ApEn } & =\lim _{N \rightarrow \infty}\left[\phi^{m}(r)-\phi^{m+1}(r)\right] \\
\text { SampEn } & =\lim _{N \rightarrow \infty}\left[-\ln \left[A^{m}(r) / B^{m}(r)\right]\right]
\end{aligned}
$$

where $r$ is the scale level; $m$, dimension with method "false nearest neighbor" [23] in which the time lag $\tau$ is obtained by autocorrelation [24]; $\phi^{m}(r), A^{m}(r), B^{m}(r)$ are of type $(N-m)^{-1} \sum_{i=1}^{N-m} \log \left(X_{i}^{m}(r)\right)$. For detailed algorithms, please refer to cited papers.

\section{Results}

To induce arrhythmia in cardiac cell culture, there are two types of methods : by injection of specific drugs such as aconitine and acetylcholine [25] or by vagal or electrical stimulation $[7,8,26]$. We are interested with the latter methods, i.e. by electrical stimulation. The culture is stimulated by a pulse train $(200 \mu \mathrm{V}$, frequency of $100 \mathrm{~Hz}$ for 5 minutes), sent by a micro-electrode located at the edge of the MEA. The pacing is carefully chosen, so that it is higher than the wave frequency in the culture in order to disrupt its electrical activities.

\section{Signal denoising}

For the method SGO, a $3^{\text {rd }}$ order filter with a sliding window of length $41 \mathrm{~ms}$ is applied to the EFP signals. In SSA, the length of basic window is 10 (unit : samples). As for method of denoising by wavelet, a multi-resolution wavelets bior3.9 from MATLAB is used.

For regular signals with slow transient, the method SGO gave a correct result for regular signals as for arrhythmic signals (Figs. 3,4). However, in case of signals with fast transient where the SGO should preserve the characteristic values (eg. peak), it failed to well reconstruct the signal (Fig. 5). In fact, the reconstructed signal lost all the peaks, In consequence, the SGO did not adapt to the EFP signals.

Applied to the same signals, the denoising by SSA showed a roughly better result than the SGO. For normal signals with slow transient, SSA worked better than method SGO. However, if the signal is very noisy, the denoised signal by SSA would be completely different from the original one (Fig. 6). Indeed, 


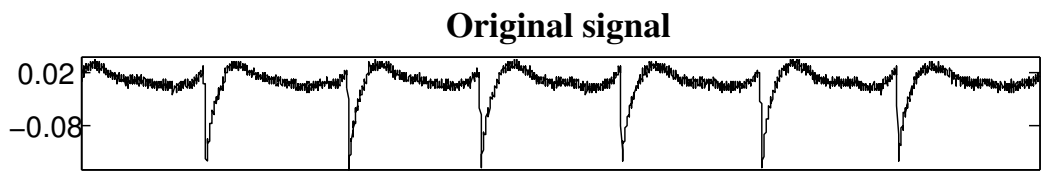

Savitzky-Golay Filtering

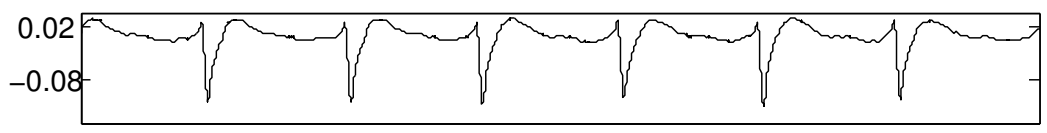

\section{Singular Spectrum Analysis}
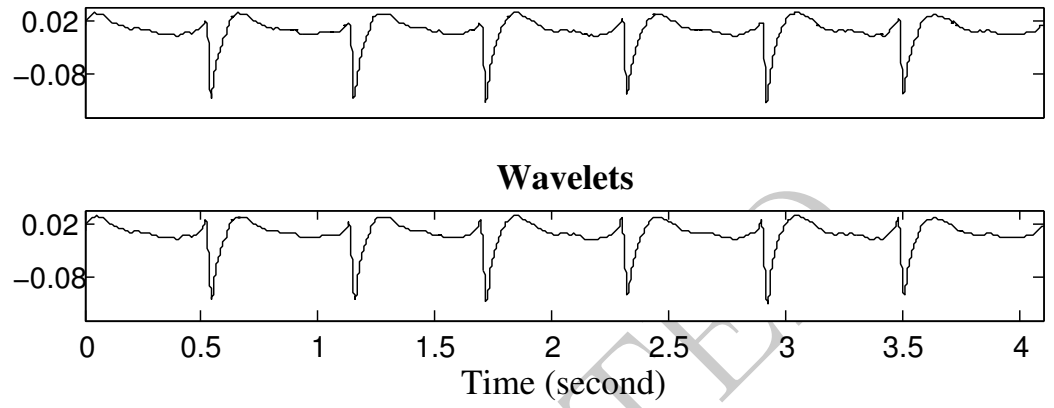

Figure 3. Comparison of three denoising methods, regular EFP signals. (y-axis : amplitude, unit : $\mathrm{mV}$ )

the SSA can help find trend in a signal. So, if the signal is too noisy, the SSA would consider that the trend is dominated by these "noise", which produces a linear reconstructed signal. In case of signal with fast transient, the result by SSA is just slightly batter than those obtained by SGO. And the important features of EFP are not preserved as well.

So a third choice is needed in order to treat all these types of signals. Compared to the previous methods, the wavelet denoising showed the best performance. Even for very noisy signal, it allows preserving a quite smoothed global shape (Fig. 6). The denoised signals are smoothed and characteristics (eg. negative / positive peaks) are preserved. So, in the pre-processing of the EFP signal, the denoising by wavelets is chosen.

\section{Complexity analysis}

As shown in Fig. 7, for EFP signals either in normal conditions or in case of arrhythmia, the entropies ApEn and SampEn are both decreasing with respect to scale $r$. For arrhythmic signals, the values of both entropies are superior to regular signals which proved that the loss of regularity in case of arrhythmia. 


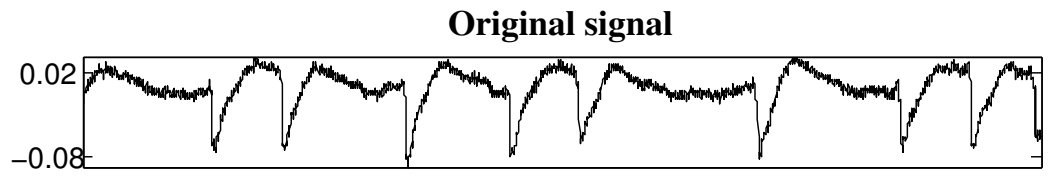

Savitzky-Golay Filtering

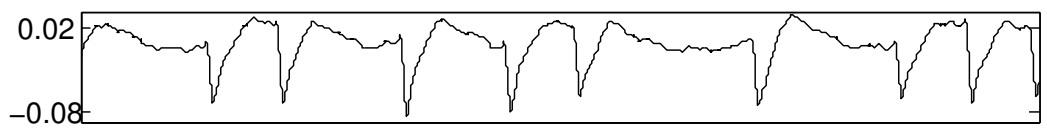

Singular Spectrum Analysis
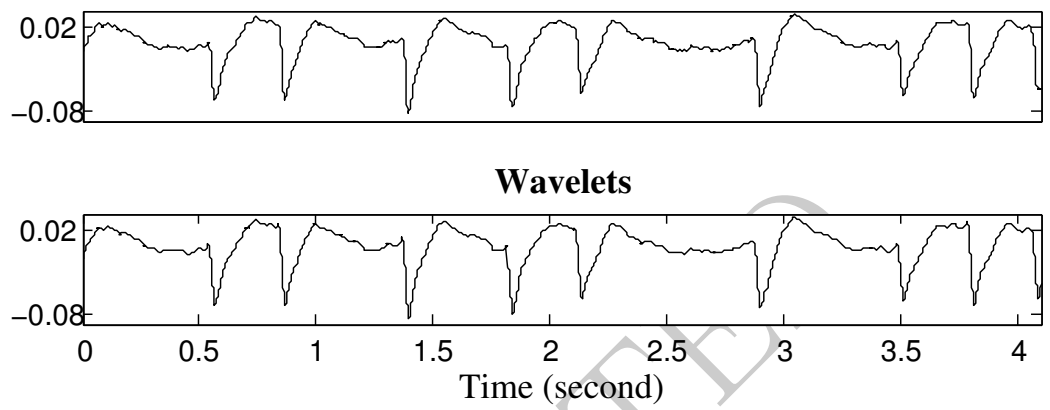

Figure 4. Comparison of three denoising methods, arrhythmic EFP signals. (y-axis : amplitude, unit : $\mathrm{mV}$ )

The typical or conventional value of $r$ is chosen as $0.2 \times$ standard derivation of the signal (arrhythmic vs. regular EFP signals, ApEn : 0.0713 vs. 0.0422 and SampEn : 0.0715 vs. 0.0422 ). So the arrhythmic EFP has almost double entropy values than those for normal signals (Table 1).

Even though these results are interesting to discriminate the regular and arrhythmic cases, the difference is not as important as visual comparison. In order to better study these signals, we performed the same Entropies study to the beats (periods) of EFP signals. One big change in case of arrhythmia of EFP signals is their periods. The regular EFP have rather stable periods regulated by intrinsic dynamics. The arrhythmia seriously disturbed this stability and made their periods no more regular (Fig. 8).

The analysis of ApEn and SampEn for periods of EFP signals are shown in Fig. 9. Different from Entropies for EFP signals themselves, the Entropies for periods of EFP signals are inversed. Not only the Entropies for regular signals have larger values, but also the orders of scale $r$ are changed. The irregular periods (Fig. 8) have some kind of "regular" dynamics which gave smaller entropies. In fact, these irregular periods are characterized by "period-doubling" as previously discussed in [8]. In case of arrhythmia, the dynamics of EFP signals would enter into chaos. The periods-doubling phenomenon is a sign when a system passed from normal state into chaos state. In this complexity analysis, the period- 

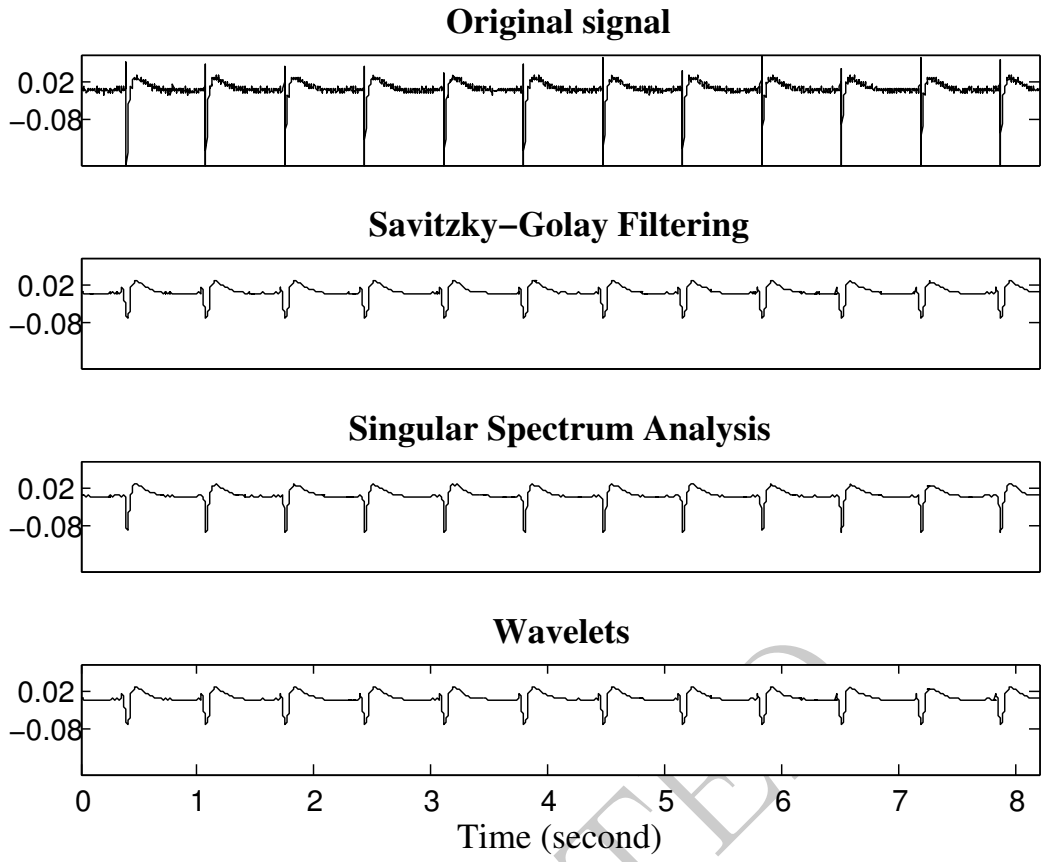

Figure 5. Comparison of three denoising methods, regular signals with fast transient. (y-axis : amplitude, unit : $\mathrm{mV}$ )

doubling phenomenon can be interpreted by a loss of 30\% - 40\% of entropies ApEn or SampEn (Table 2). If the scale $r$ is taken into account to normalize the ApEn and SampEn, when arrhythmia happens, ApEn for signals themselves are doubled and for their periods ApEn is only $7.24 \%$ of the original entropies. For SampEn, the changes are respectively 2.2591 and $8.56 \%$.

\begin{tabular}{lccccc}
\hline & \multirow{2}{c}{ ApEn } & \multicolumn{2}{c}{ SampEn } \\
& & original & normalized & original & normalized \\
\hline normal & 0.0032 & 0.0422 & 13.1875 & 0.0422 & 13.1875 \\
arrhythmic & 0.0024 & 0.0713 & 29.7083 & 0.0715 & 29.7917 \\
\hline $\begin{array}{l}\text { arrhythmic } \\
\frac{\text { arrhythmic }}{\text { normal }}\end{array}$ & & $59.19 \%$ & $44.39 \%$ & $59.02 \%$ & $44.27 \%$ \\
\hline
\end{tabular}

Table 1. Entropies values for EFP signals 


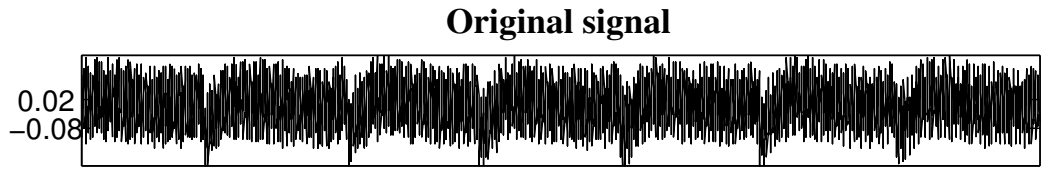

Savitzky-Golay Filtering

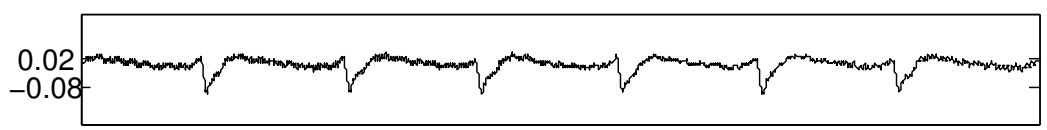

Singular Spectrum Analysis
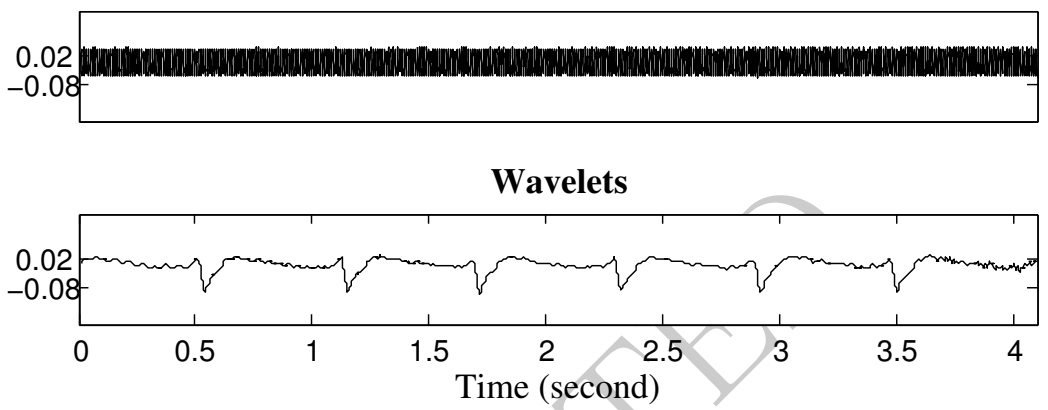

Figure 6. Comparison of three denoising methods, very noisy regular EFP signals. (y-axis : amplitude, unit : $\mathrm{mV}$ )
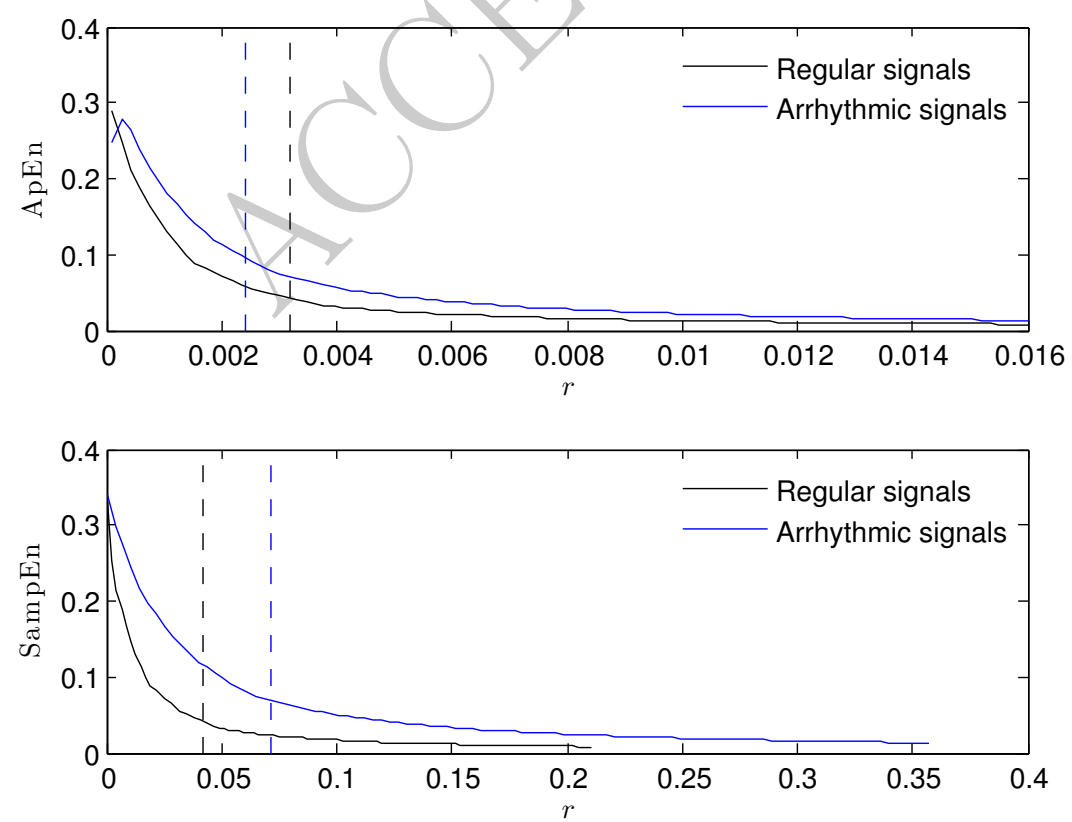

Figure 7. ApEn, SampEn for EFP signals. Dashed line indicates the conventional value of $r=0.2 \times$ standard derivation of the signal. 

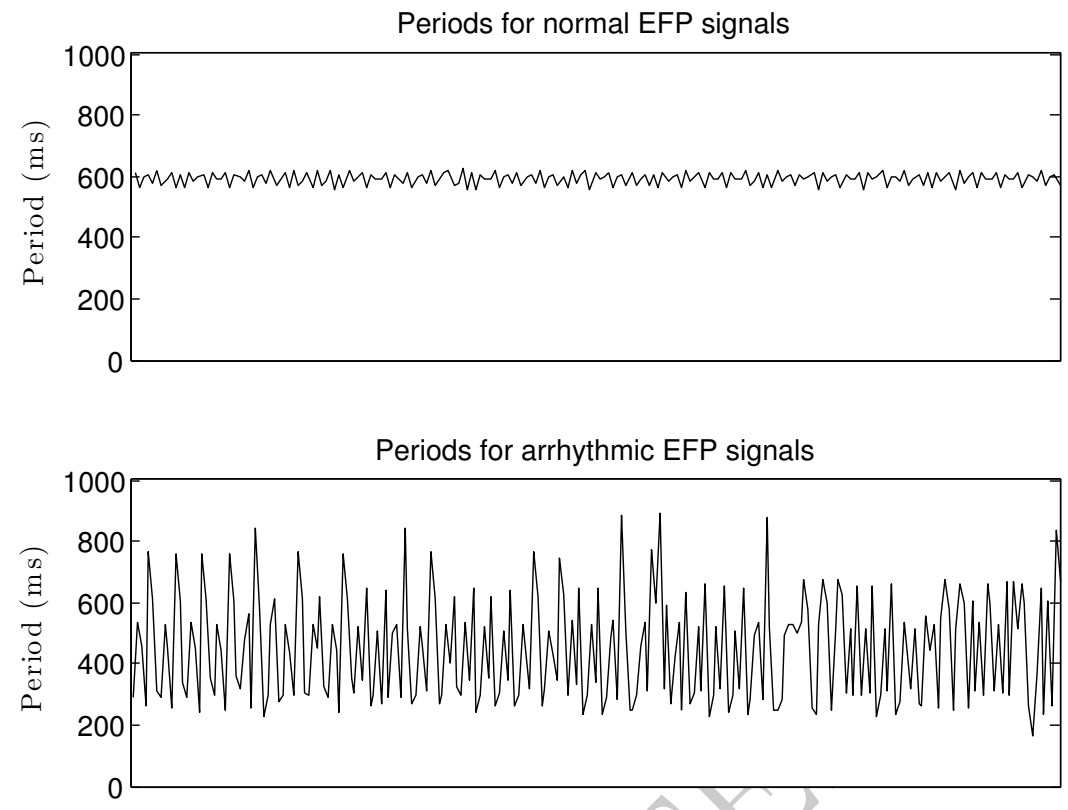

Figure 8. Periods of regular and arrhythmic EFP signals.
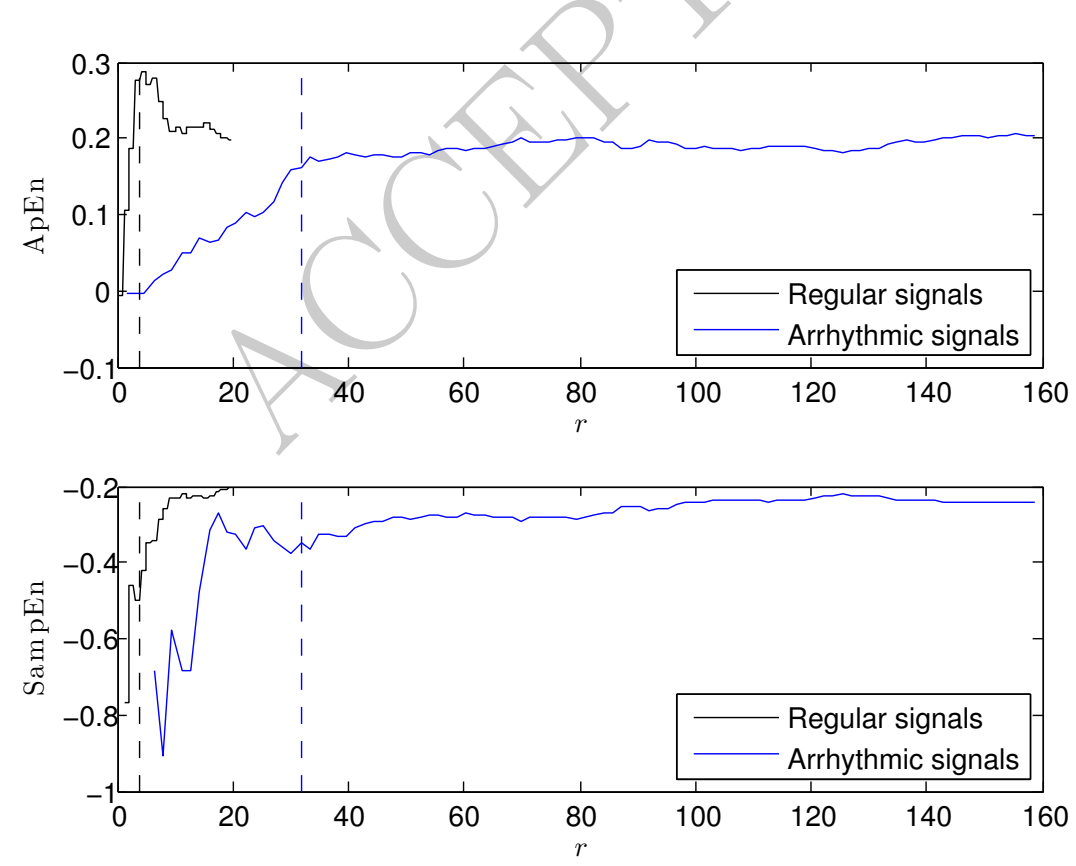

Figure 9. ApEn and SampEn for periods of EFP signals. 


\begin{tabular}{lccccc}
\hline & & \multicolumn{2}{c}{ ApEn } & \multicolumn{2}{c}{ SampEn } \\
& & original & normalized & original & normalized \\
\hline normal & 3.9151 & 0.2758 & 0.0704 & 0.4988 & 0.1274 \\
arrhythmic & 31.7406 & 0.1613 & 0.0051 & 0.3457 & 0.0109 \\
\hline $\begin{array}{c}\text { normal } \\
\text { arrhythmic }\end{array}$ & & 1.7099 & 13.8039 & 1.4429 & 11.6881 \\
\hline normal & & $58.48 \%$ & $7.24 \%$ & $69.30 \%$ & $8.56 \%$ \\
\hline
\end{tabular}

Table 2. Entropies values for periods of EFP signals

\section{Conclusions}

Cardiomyocytes culture in vitro helps to study the mechanism of cardiac diseases. Carefully prepared culture can reproduce many cardiac pathological conditions. With the platform multielectrodes array, it is possible to study these pathological conditions at cellular level. It is shown that methods based on complexity analysis allow discriminating normal signals and arrhythmic signals. In case of cardiac arrhythmia, there is an increase of Approximate Entropy and Sample Entropy $\sim 60 \%$. However, for signals' periods, the ApEn and SampEn are on the contrary 30\% - 40\% smaller for arrhythmic signals. This agreed with the period-doubling phenomenon in cardiac arrhythmia. In consequence, the ApEn and SampEn can not only serve as a discrimination index, but also provide another parameter which showed doubling phenomenon. It proves in other terms that bifurcation happens in case arrhythmia.

\section{Acknowledgements}

We acknowledge the Institute of Cardiovascular Research (Dijon, France) and the NVH Medicinal (Dr. David VANDROUX, Dijon, France) for our collaboration in the experimental data acquisition. We acknowledge the Conseil Régional de Bourgogne (Burgundy regional council) for financial support of this project.

$\mathrm{Xu}$ B. is financially supported by the Aquitanian regional council and LIRYC hospital-university research institute on cardiac rhythmology. 


\section{References}

1. W. H. Organization, ed., World Health Statistics 2012. World Health Organization, 2012.

2. M. Fejtl, A. Stett, W. Nisch, K.-H. Boven, and A. Möller, "On micro-electrode array revival: Its development, sophistication of recording, and stimulation," in Advances in Network Electrophysiology, pp. 24-37, Springer US, 2006.

3. P. Athias, D. Vandroux, C. Tissier, and L. Rochette, "Development of cardiac physiopathological models from cultured cardiomyocytes," Annales de Cardiologie et d'Angéiologie, vol. 55, pp. 90-99, apr 2006.

4. P. Athias, J. Moalic, C. Frelin, J. Klepping, and P. Padieu, "Rest and active potentials of dissociated rat heart cells in culture," Comptes rendus des séances de la Société de biologie et de ses filiales, vol. 171 , no. 1, p. 86, 1977 .

5. P. Athias, C. Frelin, B. Groz, J. Dumas, J. Klepping, and P. Padieu, "Myocardial electrophysiology: intracellular studies on heart cell cultures from newborn rats.," Pathologie-biologie, vol. 27, no. 1 , p. $13,1979$.

6. P. Athias, S. Jacquir, C. Tissier, D. Vandroux, S. Binczak, J. Bilbault, and M. Rossé, "Excitation spread in cardiac myocyte cultures using paired microelectrode and microelectrode array recordings," Journal of Molecular and Cellular Cardiology, vol. 42, no. 6, p. S3, 2007.

7. S. Jacquir, G. Laurent, D. Vandroux, S. Binczak, J. Bilbault, P. Athias, et al., "In vitro simulation of spiral waves in cardiomyocyte networks using multi-electrode array technology," Archives of cardiovascular diseases, vol. 102, no. 1, p. S63, 2009.

8. S. Jacquir, S. Binczak, B. Xu, G. Laurent, D. Vandroux, P. Athias, and J. Bilbault, "Investigation of micro spiral waves at cellular level using a microelectrode array technology," Int. J. Bifurcation Chaos, vol. 21, pp. 1-15, 2011.

9. B. Xu, S. Jacquir, S. Binczak, O. Pont, and H. Yahia, "Cardiac arrhythmia induced by hypothermia in a cardiac model in vitro," in 40th International Congress on Electrocardiology, 2013. 
10. A. Fendyur and M. E. Spira, "Towards on-chip, in-cell recordings from cultured cardiomyocytes by arrays of gold mushroom-shaped microelectrodes," Frontiers in Neuroengineering, vol. 5, pp. -, 2012.

11. M. Reppel, F. Pillekamp, Z. J. Lu, M. Halbach, K. Brockmeier, B. K. Fleischmann, and J. Hescheler, "Microelectrode arrays: A new tool to measure embryonic heart activity," Journal of Electrocardiology, vol. 37, Supplement, pp. 104-109, Oct. 2004.

12. A. Savitzky and M. J. E. Golay, "Smoothing and differentiation of data by simplified least squares procedures.," Anal. Chem., vol. 36, pp. 1627-1639, July 1964.

13. N. Golyandina, V. Nekrutkin, and A. Zhigljavsky, Analysis of time series structure: SSA and related techniques. Chapman \& Hall/CRC, 2001.

14. L. Pasti, B. Walczak, D. Massart, and P. Reschiglian, "Optimization of signal denoising in discrete wavelet transform," Chemometrics and Intelligent Laboratory Systems, vol. 48, pp. 21-34, June 1999.

15. B. N. Singh and A. K. Tiwari, "Optimal selection of wavelet basis function applied to ecg signal denoising," Digital Signal Processing, vol. 16, pp. 275-287, May 2006.

16. D. L. Donoho and J. M. Johnstone, "Ideal spatial adaptation by wavelet shrinkage," Biometrika, vol. 81 , no. 3 , pp. 425-455, 1994.

17. F. Takens, "Detecting strange attractors in turbulence," in Dynamical Systems and Turbulence, Lecture Notes in Mathematics (D. Rand and L.-S. Young, eds.), vol. 898, pp. 366-381, Springer Berlin / Heidelberg, 1981.

18. B. Xu, S. Jacquir, G. Laurent, J. Bilbault, and S. Binczak, "Phase space reconstruction of an experimental model of cardiac field potential in normal and arrhythmic conditions," in Engineering in Medicine and Biology Society (EMBC), 2013 Annual International Conference of the IEEE, 2013.

19. J.-P. Eckmann, S. O. Kamphorst, and D. Ruelle, "Recurrence plots of dynamical systems," Europhys. Lett, vol. 4, no. 9, pp. 973-977, 1987. 
20. N. Navoret, S. Jacquir, G. Laurent, and S. Binczak, "Detection of complex fractionated atrial electrograms using recurrence quantification analysis.," IEEE Trans Biomed Eng, vol. 60, pp. 19751982, Jul 2013.

21. S. M. Pincus, "Approximate entropy as a measure of system complexity." Proc Natl Acad Sci U S A, vol. 88, pp. 2297-2301, Mar 1991.

22. J. S. Richman and J. R. Moorman, "Physiological time-series analysis using approximate entropy and sample entropy," American Journal of Physiology - Heart and Circulatory Physiology, vol. 278, no. 6, pp. H2039-H2049, 2000.

23. M. B. Kennel, R. Brown, and H. D. I. Abarbanel, "Determining embedding dimension for phasespace reconstruction using a geometrical construction," Phys. Rev, A, vol. 45, pp. 3403-3411, mar 1992.

24. A. M. Albano, J. Muench, C. Schwartz, A. I. Mees, and P. E. Rapp, "Singular-value decomposition and the grassberger-procaccia algorithm," Phys. Rev. A, vol. 38, pp. 3017-3026, sep 1988.

25. M. Allessie, W. Lammers, F. Bonke, and J. Hollen, "Experimental evaluation of moe's multiple wavelet hypothesis of atrial fibrillation,") Cardiac Electrophysiology and Arrhythmias. New York: Grune ES Stratton, pp. 265-276, 1985.

26. Y. Yeh, K. Lemola, and S. Nattel, "Vagal atrial fibrillation," Acta Cardiologica Sinica, vol. 23, no. 1, pp. 1-12, 2007. 